\title{
Natural central extensions of groups
}

\author{
Christian Liedtke
}

\begin{abstract}
Given a group $G$ and an integer $n \geq 2$ we construct a new group $\widetilde{\mathcal{K}}(G, n)$. Although this construction naturally occurs in the context of finding new invariants for complex algebraic surfaces, it is related to the theory of central extensions and the Schur multiplier. A surprising application is that Abelian groups of odd order possess naturally defined covers that can be computed from a given cover by a kind of warped Baer sum.
\end{abstract}

Mathematics Subject Classification (2000). 20E22, $20 \mathrm{C} 25$.

Keywords. Covering groups, Schur multiplier, fundamental groups of plane curve complements.

\section{Introduction}

Given a group $G$ and an integer $n \geq 2$ we introduce a new group $\widetilde{\mathcal{K}}(G, n)$.

It originates from the author's work [Li] on Moishezon's programme [Mo] for complex algebraic surfaces. More precisely, to obtain finer invariants for surfaces, one attaches to a surface and an embedding into projective space the fundamental group of $\pi_{1}\left(\mathbb{P}^{2}-D\right)$, where $D$ is a curve (the branch locus of a generic projection) in the projective plane $\mathbb{P}^{2}$. Although knowing this fundamental group (and its monodromy morphism, see Section 5 for a precise statement) one can reconstruct the given surface, this fundamental group is too complicated and too large to be useful.

Instead one looks for subgroups and subquotients of this group $\pi_{1}\left(\mathbb{P}^{2}-D\right)$ to obtain the desired invariants. The most prominent one is a naturally defined subquotient that has itself a geometric interpretation, namely the fundamental group of the Galois closure of a generic projection from the given surface. Its computation already in special cases by Moishezon and Teicher [MoTe] led to counter-examples to Bogomolov's watershed conjecture.

Our construction $\widetilde{\mathcal{K}}(G, n)$ is closely related to the fundamental group of the Galois closure just mentioned (see Section 5 for an exposition). Here, we will be concerned with its group theoretical properties: 
In general, it is difficult to compute $\widetilde{\mathcal{K}}(G, n)$ for given $G$ and $n$. For example, if $G$ is Abelian we usually obtain a nilpotent group of class 2. On the other hand, $\widetilde{\mathcal{K}}(G, n)$ inherits many properties from $G$ : for example, if $G$ is finite, nilpotent, or solvable, then the same will be true for $\widetilde{\mathcal{K}}(G, n)$ and for all $n \geq 2$. This construction, defined in Section 2, is in the spirit of Hopf's computation of $H_{2}(G, \mathbb{Z})$ in terms of a presentation of $G$. And so it is not surprising there is a connection with the theory of central extensions, covers and the Schur multiplier.

Let $G$ be a finite group and $M:=M(G)$ be its Schur multiplier. Then a cover of a finite group $G$ is defined to be a group $G^{*}$, which is a central extension

$$
0 \rightarrow M \rightarrow G^{*} \rightarrow G \rightarrow 1
$$

of $M$ by $G$ such that $M$ lies in the commutator subgroup of $G^{*}$. The notion of covers comes from the study of central extensions and projective representations, see e.g. [Ka].

Every perfect group has unique cover, which is called its universal central extension. However, non-perfect groups usually do not have unique or natural covers.

If we apply our construction to an Abelian group $G$, we obtain for every $n \geq 2$ a naturally defined central extension

$$
0 \rightarrow M(G) \rightarrow \tilde{\mathcal{K}}(G, n) \rightarrow G^{n-1} \rightarrow 1
$$

If $n \geq 3$ or if $n=2$ and $G$ has odd order, then $M(G)$ lies in the centre of $\tilde{\mathcal{K}}(G, n)$.

A surprising application (the case $n=2$ ) is the existence of natural covers for Abelian groups of odd order. This natural cover can be computed from a given cover by a sort of warped Baer sum of this given cover with itself.

This article is organised as follows.

In Section 1 we construct the auxiliary group $\mathcal{K}(G, n)$ for $n \geq 2$ and a given group $G$. It is a subgroup of $G^{n}$ and not so difficult to compute, especially when $G$ is perfect or Abelian.

In Section 2 we use this auxiliary construction to define $\widetilde{\mathcal{K}}(G, n)$, the main object of this article. We show that it is a central extension of $H_{2}(G, \mathbb{Z})$ by $\mathcal{K}(G, n)$. Also, we prove that it inherits properties such as finiteness, nilpotency or solvability from $G$.

In Section 3 we prove that $\widetilde{\mathcal{K}}(G, n)$ can be computed from an arbitrary cover of $G$. In particular, it contains the universal central extension of $G$ in case $G$ is perfect.

In Section 4 we determine the structure, i.e. the centre, Frattini and commutator subgroup, of $\widetilde{\mathcal{K}}(G, n)$ in case $G$ is Abelian. Here we also prove that Abelian groups of odd order possess natural covers.

In Section 5 we describe the relation to fundamental groups of algebraic surfaces and to Moishezon's programme to find finer invariants for surfaces.

Acknowledgements. This article extends results from my Ph.D. thesis [Li]. I thank my supervisor Gerd Faltings for discussions and help, as well as the Max-Planck- 
Institut in Bonn for hospitality and financial support. Also, I thank Irene Bouw for proofreading and many suggestions as well as the referee for pointing out inaccuracies.

\section{An auxiliary construction}

We let $G$ be a group and $n \geq 2$ a natural number. We denote by $G^{\mathrm{ab}}:=G /[G, G]$ the Abelianisation of $G$. Then we define a map

$$
\begin{aligned}
\psi: G^{n} & \rightarrow G^{\mathrm{ab}}, \\
\left(g_{1}, \ldots, g_{n}\right) & \mapsto g_{1} \ldots g_{n}[G, G],
\end{aligned}
$$

which is a homomorphism as $G^{\mathrm{ab}}$ is Abelian.

Definition 1.1. For a group $G$ and a natural number $n \geq 2$ we define $\mathcal{K}(G, n)$ to be the kernel of the homomorphism $\psi: G^{n} \rightarrow G^{\mathrm{ab}}$.

Clearly $\mathcal{K}(-, n)$ is functorial in its first argument. We start with the

Proposition 1.2. Let $G_{1}, G_{2}, G$ be arbitrary groups and $n \geq 2$ a natural number.

(1) If $G_{1} \rightarrow G_{2}$ is an injective or a surjective homomorphism then the same is true for the induced maps $\mathcal{K}\left(G_{1}, n\right) \rightarrow \mathcal{K}\left(G_{2}, n\right)$.

(2) There exists a natural isomorphism $\mathcal{K}\left(G_{1} \times G_{2}, n\right) \cong \mathcal{K}\left(G_{1}, n\right) \times \mathcal{K}\left(G_{2}, n\right)$.

(3) For $n \geq 3$ the natural homomorphism from $\mathcal{K}(G, n)^{\mathrm{ab}}$ onto $\mathcal{K}\left(G^{\mathrm{ab}}, n\right)$ is an isomorphism.

Proof. The first two assertions follow immediately from the definition.

The surjection $G \rightarrow G^{\mathrm{ab}}$ and the universal property of the Abelianisation imply that there is a natural surjective homomorphism $\mathcal{K}(G, n)^{\mathrm{ab}} \rightarrow \mathcal{K}\left(G^{\mathrm{ab}}, n\right)$. An element of the kernel $\mathcal{K}(G, n) \rightarrow \mathcal{K}\left(G^{\mathrm{ab}}, n\right)$ is also an element of the kernel $G^{n} \rightarrow\left(G^{\mathrm{ab}}\right)^{n}$, which is $[G, G]^{n}$. Since we assumed that $n \geq 3$, we may write

$$
\left(\left[h_{1}, h_{2}\right], 1, \ldots, 1\right)=\left[\left(h_{1}, h_{1}^{-1}, 1, \ldots, 1\right),\left(h_{2}, 1, h_{2}^{-1}, \ldots, 1\right)\right] \in G^{n} .
$$

Thus $[G, G]^{n}$ is not only a subgroup of $\mathcal{K}(G, n)$ but also lies inside the commutator subgroup of $\mathcal{K}(G, n)$. Hence the kernel $\mathcal{K}(G, n) \rightarrow \mathcal{K}\left(G^{\mathrm{ab}}, n\right)$ is the commutator subgroup of $\mathcal{K}(G, n)$, and we are done.

Remark 1.3. Already here we see that the case $n=2$ has to be treated separately. If we need $n \geq 3$ for a statement it is usually easy to obtain a counter-example for the corresponding statement for $n=2$ using elementary Abelian 2-groups, dihedral groups or the quaternion group. 
In the following two cases it is particularly easy to determine $\mathcal{K}(G, n)$.

Proposition 1.4. (1) If $G$ is perfect then $\mathcal{K}(G, n) \cong G^{n}$.

(2) If $G$ is Abelian then $\mathcal{K}(G, n) \cong G^{n-1}$. This isomorphism is not canonical.

Proof. The Abelianisation of a perfect group is trivial, and so the first assertion follows from the definition of $\mathcal{K}(G, n)$.

Now let $G$ be an Abelian group. Then the map

$$
\begin{aligned}
G^{n-1} & \rightarrow G^{n}, \\
\left(g_{1}, \ldots, g_{n-1}\right) & \mapsto\left(g_{1}, \ldots, g_{n-1},\left(g_{1} \ldots g_{n-1}\right)^{-1}\right),
\end{aligned}
$$

defines a homomorphism. It is injective with image $\mathcal{K}(G, n)$.

Proposition 1.5. Let $n \geq 2$ and let $P$ be one of the following properties:

Abelian, finite, nilpotent, perfect, solvable.

Then $G$ has the property $P$ if and only if $\mathcal{K}(G, n)$ has the same property.

Proof. By definition, $\mathcal{K}(G, n)$ is a subgroup of $G^{n}$. Therefore if $G$ is Abelian (resp. finite, nilpotent, solvable) the same is true for $\mathcal{K}(G, n)$. If $G$ is perfect then $\mathcal{K}(G, n)=G^{n}$, which is also perfect.

The projection onto the first factor $G^{n} \rightarrow G$ induces a surjective homomorphism from $\mathcal{K}(G, n)$ onto $G$. Hence $G$ is a quotient of $\mathcal{K}(G, n)$. Therefore if $\mathcal{K}(G, n)$ is Abelian (resp. finite, nilpotent, perfect, solvable) the same is true for $G$.

\section{The main construction}

As in the previous section, we let $G$ be a group and $n \geq 2$ be a natural number. We choose a presentation $G \cong F / N$ where $F$ is a free group. Then $\mathcal{K}(N, n)$ is a subgroup of $\mathcal{K}(F, n)$ which is a subgroup of $F^{n}$.

We denote by $\langle\langle\mathcal{K}(N, n)\rangle\rangle$ the subgroup normally generated by $\mathcal{K}(N, n)$ inside $F^{n}$. For $n \geq 3$, it is not difficult to see (using formula (1)) that the normal closure $\left\langle\langle\mathcal{K}(N, n)\rangle\right.$ of $\mathcal{K}(N, n)$ inside $F^{n}$ is equal to the normal closure of $\mathcal{K}(N, n)$ inside $\mathcal{K}(F, n)$.

Definition 2.1. We let $G$ be a group and $n \geq 2$ be a natural number. We define

$$
\tilde{\mathcal{K}}(G, n):=\mathcal{K}(F, n) /\langle\langle\mathcal{K}(N, n)\rangle .
$$


Theorem 2.2. The group $\widetilde{\mathcal{K}}(G, n)$ does not depend upon the choice of a presentation. There exists a central short exact sequence

$$
0 \rightarrow H_{2}(G, \mathbb{Z}) \rightarrow \tilde{\mathcal{K}}(G, n) \rightarrow \mathcal{K}(G, n) \rightarrow 1
$$

For $n \geq 3$ the group $\mathrm{H}_{2}(G, \mathbb{Z})$ lies inside the commutator subgroup of $\tilde{\mathcal{K}}(G, n)$.

Proof. We choose a presentation $G \cong F / N$ and abbreviate the normal closure $\langle\langle\mathcal{K}(N, n)\rangle\rangle$ of $\mathcal{K}(N, n)$ in $F^{n}$ by $R$.

First, we will prove the short exact sequence of the statement of the theorem: Let $\pi$ be the projection of $F^{n}$ onto its last $n-1$ factors. By abuse of notation we denote its restriction to $\mathcal{K}(F, n)$ again by $\pi$. We obtain a short exact sequence

$$
1 \rightarrow[F, F] \rightarrow \mathcal{K}(F, n) \stackrel{\pi}{\rightarrow} F^{n-1} \rightarrow 1 .
$$

An easy computation with commutators shows that $R \cap \operatorname{ker} \pi=[F, N]$. Via $\pi$ we obtain the following diagram with exact rows and columns:

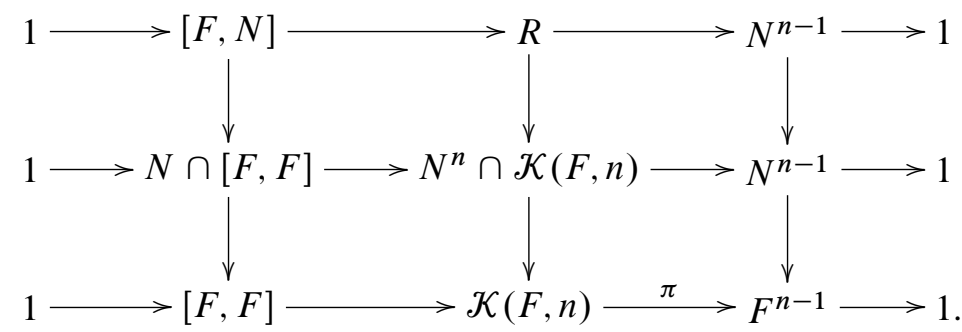

Taking quotients of successive rows we exhibit $\mathcal{K}(F, n) / R$ as an extension of $(N \cap$ $[F, F]) /[F, N]$ by $\mathcal{K}(F, n) /\left(N^{n} \cap \mathcal{K}(F, n)\right)$. The latter group is isomorphic to $\mathcal{K}(G, n)$. By Hopf's theorem (cf. [Br, Theorem II.5.3]), the group $(N \cap[F, F]) /[F, N]$ is isomorphic to $\mathrm{H}_{2}(G, \mathbb{Z})$. Hence we obtain an extension

$$
1 \rightarrow H_{2}(G, \mathbb{Z}) \rightarrow \mathcal{K}(F, n) / R \rightarrow \mathcal{K}(G, n) \rightarrow 1 .
$$

Next, we will show that this extension is central: Every element of $H_{2}(G, \mathbb{Z})$ can be lifted to an element of the form $\vec{x}:=(x, 1, \ldots, 1)$ of $\mathcal{K}(F, n)$ with $x \in N \cap[F, F]$. For $\vec{y}:=\left(y_{1}, \ldots, y_{n}\right) \in \mathcal{K}(F, n)$ we compute

$$
\vec{y} \vec{x} \vec{y}^{-1}=(\underbrace{\left[y_{1}, x\right]}_{\in[F, N]}, 1, \ldots, 1) \cdot(x, 1, \ldots, 1) \equiv \vec{x} \quad \bmod [F, N] .
$$

Hence $H_{2}(G, \mathbb{Z})$ lies inside the centre of $\mathcal{K}(F, n) / R$.

We now prove that $\widetilde{\mathcal{K}}(G, n)$ is well defined: Let $\alpha: F / N \cong F^{\prime} / N^{\prime}$ be another presentation of $G$. We lift this isomorphism to a map $\varphi: F \rightarrow F^{\prime}$. Then $\varphi$ maps $N$ 
to $N^{\prime}$ and hence $\mathcal{K}(N, n)$ to $\mathcal{K}\left(N^{\prime}, n\right)$. Let $R^{\prime}$ be the normal closure of $\mathcal{K}\left(N^{\prime}, n\right)$ inside $F^{\prime n}$. Then $\varphi$ induces a homomorphism

$$
\bar{\varphi}: \mathcal{K}(F, n) / R \rightarrow \mathcal{K}\left(F^{\prime}, n\right) / R^{\prime} .
$$

We let $\varphi^{\prime}$ be another map lifting $\alpha$ to a homomorphism from $F$ to $F^{\prime}$.

Suppose now that $n \geq 3$. Then elements of the form $\left(1, \ldots, f, 1, \ldots, f^{-1}, 1, \ldots\right)$ generate $\mathcal{K}(F, n)$ and so in this case it is enough to compare the maps induced by $\varphi$ and $\varphi^{\prime}$ on such elements. For $f \in F$ there exists $n_{f}^{\prime} \in N^{\prime}$ such that $\varphi(f)=\varphi^{\prime}(f) n_{f}^{\prime}$. Hence

$$
\begin{aligned}
\varphi\left(\left(f, f^{-1}, \ldots\right)\right) & =\left(\varphi^{\prime}(f) n_{f}^{\prime}, n_{f}^{-1} \varphi^{\prime}(f)^{-1}, \ldots\right) \\
& =\varphi^{\prime}\left(\left(f, f^{-1}, \ldots\right)\right) \cdot \underbrace{\left(n_{f}^{\prime}, \varphi^{\prime}(f) n_{f}^{-1} \varphi^{\prime}(f)^{-1}, \ldots\right)}_{\in R^{\prime}} .
\end{aligned}
$$

Hence the induced maps coincide. For $n=2$, the group $\mathcal{K}(F, 2)$ is generated by elements of the form $\left(f, f^{-1}\right)$ and $\left(\left[f_{1}, f_{2}\right], 1\right)$. It is easy to see that also in this case the induced maps coincide.

In particular, if we choose $F=F^{\prime}$ and $N=N^{\prime}$ with $\alpha$ and $\varphi$ the identity then every other lift $\varphi^{\prime}$ of the identity induces the identity on $\mathcal{K}(F, n) / R$.

Coming back to the general case, we let $F / N$ and $F^{\prime} / N^{\prime}$ be two presentations of $G$ and let $\alpha$ be an isomorphism between them. Then $\alpha$ and $\alpha^{-1}$ induce maps between $\mathcal{K}(F, n) / R$ and $\mathcal{K}\left(F^{\prime}, n\right) / R^{\prime}$ such that the composites of these induced maps have to be the identity by the previous paragraph. Hence $\alpha$ induces an isomorphism from $\mathcal{K}(F, n) / R$ to $\mathcal{K}\left(F^{\prime}, n\right) / R^{\prime}$. Thus, $\widetilde{\mathcal{K}}(G, n)$ is well defined.

Taking the quotient of the top row by the bottom row of (3) we obtain a short exact sequence

$$
1 \rightarrow[F, F] /[F, N] \rightarrow \mathcal{K}(F, n) / R \rightarrow G^{n-1} \rightarrow 1 .
$$

The inclusion of $H_{2}(G, \mathbb{Z})$ into $\mathcal{K}(F, n) / R$ factors over $[F, F] /[F, N]$. Suppose now that $n \geq 3$. Then $[F, F]$ lies inside the commutator subgroup of $\mathcal{K}(F, n)$, cf. formula (1). Hence $\mathrm{H}_{2}(G, \mathbb{Z})$ lies inside the commutator subgroup of $\mathcal{K}(F, n) / R$.

Corollary 2.3. A homomorphism $\alpha: G \rightarrow H$ induces a map $\widetilde{\mathcal{K}}(G, n) \rightarrow \widetilde{\mathcal{K}}(H, n)$.

The short exact sequence (2) induces maps $H_{2}(G, \mathbb{Z}) \rightarrow H_{2}(H, \mathbb{Z})$ and $\mathcal{K}(G, n) \rightarrow \mathcal{K}(H, n)$. These maps coincide with the map induced by $\alpha$ on homology and the map induced by a from $\mathcal{K}(G, n)$ to $\mathcal{K}(H, n)$, respectively.

Proof. We choose presentations $G \cong F / N$ and $H \cong F^{\prime} / N^{\prime}$. In the proof of Theorem 2.2 we did not need that the map $\alpha$ considered there was an isomorphism to prove that it induces a unique map from $\widetilde{\mathcal{K}}(G, n)$ to $\widetilde{\mathcal{K}}(H, n)$. This shows functoriality. 
It is easy to see that the induced map coming from $\tilde{\mathcal{K}}(-, n)$ is compatible with the map induced by $\alpha$ from $\mathcal{K}(G, n)$ to $\mathcal{K}(H, n)$.

We have to prove that the homomorphism induced on homology is compatible with the one coming from $\widetilde{\mathcal{K}}(-, n)$. However, this follows from [Br], Exercise II.6.3.b.

Corollary 2.4. For $n \geq 3$ there exist isomorphisms

$$
\tilde{\mathcal{K}}(G, n)^{\mathrm{ab}} \cong \mathcal{K}(G, n)^{\mathrm{ab}} \cong \mathcal{K}\left(G^{\mathrm{ab}}, n\right) \cong\left(G^{\mathrm{ab}}\right)^{n-1} .
$$

Proof. The first isomorphism follows from the fact that $H_{2}(G, \mathbb{Z})$ lies inside the commutator subgroup of $\widetilde{\mathcal{K}}(G, n)$. The remaining isomorphisms follow from Proposition 1.2 and Proposition 1.4.

Corollary 2.5. If $G$ is cyclic then $\widetilde{\mathcal{K}}(G, n) \cong G^{n-1}$. This isomorphism is not canonical.

Proof. If $G$ is cyclic then $H_{2}(G, \mathbb{Z})$ vanishes. Hence $\widetilde{\mathcal{K}}(G, n)$ is isomorphic to $\mathcal{K}(G, n)$, which is isomorphic to $G^{n-1}$ by Proposition 1.4.

Proposition 2.6. Let $P$ be one of the following properties:

finite, nilpotent, perfect, solvable.

Then $G$ has the property $P$ if and only if $\widetilde{\mathcal{K}}(G, n)$ has the same property.

Proof. If $G$ is finite then so are $H_{2}(G, \mathbb{Z})$ and $\mathcal{K}(G, n)$. Hence $\widetilde{\mathcal{K}}(G, n)$ is finite because it is an extension of $H_{2}(G, \mathbb{Z})$ by $\mathcal{K}(G, n)$.

Since $\mathrm{H}_{2}(G, \mathbb{Z})$ is Abelian it is nilpotent. Hence if $G$ is solvable (resp. nilpotent) then so is $\widetilde{\mathcal{K}}(G, n)$ because it is a (central) extension of two solvable (resp. nilpotent) groups.

If $G$ is perfect and $G \cong F / N$ then also $\widetilde{G}:=[F, F] /[F, N]$ is perfect. By the short exact sequence (4) the group $\widetilde{\mathcal{K}}(G, n)$ is an extension of $\widetilde{G}$ by $G^{n-1}$. Thus $\widetilde{\mathcal{K}}(G, n)$ is perfect being an extension of two perfect groups.

The group $G$ is a quotient of $\widetilde{\mathcal{K}}(G, n)$. So, if $\widetilde{\mathcal{K}}(G, n)$ is finite (resp. nilpotent, perfect, solvable) the same is true for $G$.

We end this section by a remark on group actions on $\mathcal{K}(-, n)$ and $\widetilde{\mathcal{K}}(-, n)$.

Given a group $F$, the symmetric group $\widetilde{S}_{n}$ on $n$ letters acts on $F^{n}$ by permuting its $n$ factors. Clearly, this action preserves $\mathcal{K}(F, n)$. It is not difficult to see that if $G \cong F / N$ is a presentation of $G$, then the $\Im_{n}$-action on $\mathcal{K}(F, n)$ induces a $\Im_{n}$-action on $\widetilde{\mathcal{K}}(G, n)$ that does not depend on the choice of a presentation of $G$.

We let $\widetilde{S}_{n-1}$ be the subgroup of $\widetilde{S}_{n}$ of those permutations that fix, say, the first letter. Inside $\mathcal{K}(G, n)$ (resp. $\widetilde{\mathcal{K}}(G, n))$ we form the normal closure $N$ (resp. $\tilde{N}$ ) 
of the subgroup generated by the elements $g \cdot \sigma\left(g^{-1}\right)$, for all $\sigma \in \widetilde{S}_{n-1}$ and all $g \in \mathcal{K}(G, n)($ resp. $g \in \widetilde{\mathcal{K}}(G, n))$. Then the quotients $\mathcal{K}(G, n) / N$ and $\widetilde{\mathcal{K}}(G, n) / \widetilde{N}$ are isomorphic to $G$.

Thus, the $\widetilde{S}_{n}$-actions on $\mathcal{K}(G, n)$ and $\widetilde{\mathcal{K}}(G, n)$ allow us to recover $G$ as a quotient of these groups. Although we do not need this result here, it is crucial in the context of the geometric origin of these groups. We refer to [Li], Section 5, for proofs.

\section{Central extensions and covers}

We recall that a group $G^{*}$ is called a cover (or a representation group) of the finite group $G$ if there exists a central short exact sequence

$$
0 \rightarrow M \rightarrow G^{*} \rightarrow G \rightarrow 1
$$

with $M \leq\left[G^{*}, G^{*}\right]$ and such that $M$ is isomorphic to the Schur multiplier of $G$. For a perfect group there exists a unique cover up to isomorphism, which is called its universal central extension.

If $G$ is finite then Pontryagin duality provides us with a non-canonical isomorphism of its Schur multiplier $M(G):=H^{2}\left(G, \mathbb{C}^{*}\right)$ with $H_{2}(G, \mathbb{Z})$.

Proposition 3.1. If $G$ is a finite and perfect group there exists a short exact sequence

$$
1 \rightarrow \tilde{G} \rightarrow \tilde{\mathcal{K}}(G, n) \rightarrow G^{n-1} \rightarrow 1 .
$$

Here $\widetilde{G}$ denotes the universal central extension of $G$.

Proof. If $G$ is perfect with presentation $F / N$ then its universal central extension is isomorphic to $[F, F] /[F, N]$, cf. [Ka], Theorem 2.10.3. The statement follows from the short exact sequence (4).

This result suggests that there is a connection of $\widetilde{\mathcal{K}}(-, n)$ with the theory of central extensions. This is in fact true by the following theorem which tells us that we can compute $\tilde{\mathcal{K}}(G, n)$ using an arbitrary cover of $G$.

Theorem 3.2. Let $G^{*}$ be a cover of the finite group $G$ and $M$ be the kernel of the map from $G^{*}$ onto $G$. For $n \geq 2$ there exists an isomorphism

$$
\mathcal{K}\left(G^{*}, n\right) / \mathcal{K}(M, n) \cong \tilde{\mathcal{K}}(G, n) .
$$

In particular, the group on the left depends on $G$ and $n$ only. 
Proof. By Schur's theorem [Ka], Theorem 2.4.6, there exists a free group $F$ and two normal subgroups $N$ and $S$ such that $G \cong F / N, G^{*} \cong F / S$ and $N /[F, N]=$ $S /[F, N] \times(N \cap[F, F]) /[F, N]$.

First, we show that

$$
H:=\left\langle\mathcal{K}(F, n) \cap S^{n}, \mathcal{K}(N, n)\right\rangle
$$

is a normal subgroup of $F^{n}$ contained in $\mathcal{K}(F, n)$. Since both $S^{n}$ and $\mathcal{K}(F, n)$ are normal in $F^{n}$ we see that $\mathcal{K}(F, n) \cap S^{n}$ is a normal subgroup of $F^{n}$ contained in $\mathcal{K}(F, n)$. This already shows that $H$ is a subgroup of $\mathcal{K}(F, n)$. To show normality in $F^{n}$ it is enough to show that conjugates of $\mathcal{K}(N, n)$ by elements of $F^{n}$ lie inside $H$. So let $\left(x_{1}, \ldots, x_{n}\right) \in \mathcal{K}(N, n)$ and $f \in F$. Then

$$
(f, 1, \ldots)\left(x_{1}, \ldots, x_{n}\right)(f, 1, \ldots)^{-1}=\underbrace{\left(\left[f, x_{1}\right], 1, \ldots\right)}_{\in[F, N]} \cdot \underbrace{\left(x_{1}, \ldots, x_{n}\right)}_{\in \mathcal{K}(N, n)} .
$$

By Schur's theorem mentioned in the beginning, $[F, N]$ is contained in $S$ and it is straight forward to see that $[F, N]^{n}$ is contained in $\mathcal{K}(F, n) \cap S^{n}$ (both regarded as subgroups of $F^{n}$ ). Hence this conjugate lies in $H$ and from this calculation it is easy to deduce the normality of $H$ in $F^{n}$.

We already mentioned that $\mathcal{K}(F, n) \cap S^{n}$ is a normal subgroup of $F^{n}$ contained in $\mathcal{K}(F, n)$ and from the presentation $G^{*} \cong F / S$ we easily get an isomorphism

$$
\mathcal{K}\left(G^{*}, n\right) \cong \mathcal{K}(F, n) /\left(\mathcal{K}(F, n) \cap S^{n}\right) .
$$

Since the image of $N$ in $F / S \cong G^{*}$ is $M$, we see that $\mathcal{K}(N, n)$ maps to $\mathcal{K}(M, n)$ inside $\mathcal{K}\left(G^{*}, n\right)$ under the isomorphism (5). $\mathcal{K}(M, n)$ is generated by elements of the form $\left(1, \ldots, m, 1, \ldots, m^{-1}, 1, \ldots\right)$ with $m \in M$, which is also true for $n=2$ since $M$ is Abelian. Such elements of $\mathcal{K}\left(G^{*}, n\right)$ can be lifted to elements of $\mathcal{K}(F, n)$ lying inside $\mathcal{K}(N, n)$. Hence $\mathcal{K}(N, n)$ maps surjectively onto $\mathcal{K}(M, n)$.

Putting these results together we obtain an isomorphism

$$
\mathcal{K}\left(G^{*}, n\right) / \mathcal{K}(M, n) \cong \mathcal{K}(F, n) / H .
$$

By definition, the quotient of $\mathcal{K}(F, n)$ by the normal closure $\langle\langle\mathcal{K}(N, n)\rangle\rangle$ of $\mathcal{K}(N, n)$ inside $F^{n}$ is $\mathcal{K}(G, n)$. Clearly, $\mathcal{K}(N, n)$ is contained in $H$ and since $H$ is normal in $F^{n}$, also $\langle\langle\mathcal{K}(N, n)\rangle\rangle$ is contained in $H$. Hence the inclusion of these two normal subgroups induces a surjective homomorphism

$$
\psi: \tilde{\mathcal{K}}(G, n) \rightarrow \mathcal{K}\left(G^{*}, n\right) / \mathcal{K}(M, n) .
$$

On the other hand, both groups are extensions of $H_{2}(G, \mathbb{Z})$ by $\mathcal{K}(G, n)$. The surjective map $\psi$ induces an isomorphism between the kernel and the quotient of these extensions. Hence $\psi$ is an isomorphism. 


\section{Abelian groups}

We will now see that already in the case of finite Abelian groups it is quite difficult to determine the structure of $\widetilde{\mathcal{K}}(G, n)$. Thanks to Proposition 1.4, we know that $\mathcal{K}(G, n)$ is isomorphic to $G^{n-1}$.

The following proposition implies that we may restrict ourselves to $p$-groups:

Proposition 4.1. Let $G$ be a finite nilpotent group and $n \geq 2$. Let $S_{p}$ be its unique Sylow p-subgroup. There exists an isomorphism

$$
\tilde{\mathcal{K}}(G, n) \cong \prod_{p} \tilde{\mathcal{K}}\left(S_{p}, n\right)
$$

where $p$ runs over all prime numbers. More precisely, the short exact sequence (2) for $G$ is the product of the short exact sequences (2) taken over all its Sylow p-subgroups $S_{p}$.

Proof. By functoriality, there exists a commutative diagram with exact rows:

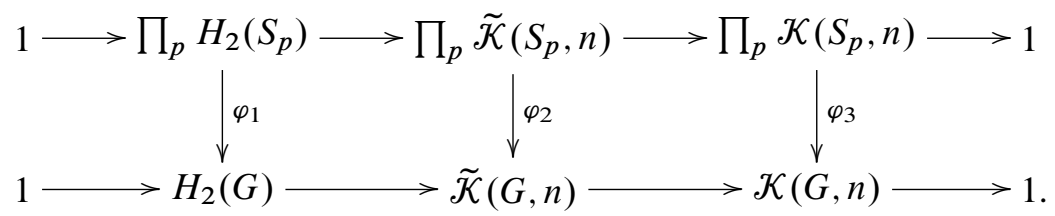

From [Ka], Corollary 2.2.11, and Proposition 1.2 it follows that $\varphi_{1}$ and $\varphi_{3}$ are isomorphisms. Hence $\varphi_{2}$ is an isomorphism.

First, we deal with $n=2$, which is the most interesting case from the point of view of group theory. Since $G$ is Abelian, $\mathcal{K}(G, 2)$ is isomorphic to $G$ and (2) becomes

$$
0 \rightarrow H_{2}(G, \mathbb{Z}) \rightarrow \tilde{\mathcal{K}}(G, 2) \rightarrow G \rightarrow 1 .
$$

Proposition 4.2. Let $G$ be a finite Abelian p-group.

(1) If $p \neq 2$ then $\widetilde{\mathcal{K}}(G, 2)$ is a cover of $G$ via the short exact sequence (6).

(2) If $G$ is an elementary Abelian 2-group then $\tilde{\mathcal{K}}(G, 2)$ is an elementary Abelian 2-group. More precisely, it is the product of $G$ and $\mathrm{H}_{2}(G, \mathbb{Z})$.

Proof. Let $G$ be an Abelian $p$-group with $p \neq 2$. Every cover of $G$ is nilpotent of class at most 2 . The same is true for $\widetilde{\mathcal{K}}(G, 2)$ by $(6)$. Hence for arbitrary elements in these groups the commutator relation $\left[x^{i}, y^{j}\right]=[x, y]^{i j}$ holds true.

To prove our statement we have to show that $H_{2}(G, \mathbb{Z})$ in (6) lies in the commutator subgroup of $\widetilde{\mathcal{K}}(G, 2)$. We choose an arbitrary cover $G^{*} \rightarrow G$ with kernel $M$ and 
exhibit $\widetilde{\mathcal{K}}(G, 2)$ as in Theorem 3.2. It is enough to prove that $\langle(m, 1) \mid m \in M\rangle$ lies inside the commutator subgroup of $\mathcal{K}\left(G^{*}, 2\right)$ modulo elements of $\mathcal{K}(M, 2)$

Since $G$ is Abelian, $M$ coincides with the commutator subgroup of $G^{*}$ and so we have to check that the commutator subgroup of $G^{*}$ is a subgroup of the commutator subgroup of $\mathcal{K}\left(G^{*}, 2\right)$ modulo elements of $\mathcal{K}(M, 2)$. Given $x, y \in G^{*}$, the elements $\left(x, x^{-1}\right)$ and $\left(y, y^{-1}\right)$ lie in $\mathcal{K}\left(G^{*}, 2\right)$ and hence $\left([x, y],\left[x^{-1}, y^{-1}\right]\right)$ lies inside the commutator subgroup of $\mathcal{K}\left(G^{*}, 2\right)$. Modulo $\mathcal{K}(M, 2)$ this element is congruent to $\left([x, y]^{2}, 1\right)$.

Since $G$ has odd order, also $M$ has odd order by Schur's theorem, cf. [Ka], Theorem 2.1.5. Hence $([x, y], 1)$ is a power of $\left([x, y]^{2}, 1\right)$. Thus, modulo elements of $\mathcal{K}(M, 2)$, the element $([x, y], 1)$ lies in the commutator subgroup of $\mathcal{K}\left(G^{*}, 2\right)$.

Now, let $G$ be an elementary Abelian 2-group. To prove the remaining statement we can either proceed as above or we copy the first part of the proof of Proposition 4.7 below.

Definition 4.3. Let $G$ be an Abelian group. We say that $G$ has a natural cover if $\widetilde{\mathcal{K}}(G, 2)$ is a cover of $G$. In this case, we will also refer to $\widetilde{\mathcal{K}}(G, 2)$ as the natural cover of $G$.

The following result is an immediate corollary of the previous proposition.

Theorem 4.4. A finite Abelian group of odd order possesses a natural cover.

More precisely, let $G^{*}$ be an arbitrary cover of an Abelian group $G$ of odd order and let $M$ be the kernel of $G^{*} \rightarrow G$. Then we obtain the natural cover of $G$ as a subquotient of $\left(G^{*}\right)^{2}$ via

$$
\widetilde{\mathcal{K}}(G, 2) \cong\left\langle\left(g, g^{-1}\right),(m, 1) \mid g \in G^{*}, m \in M\right\rangle /\left\langle\left(m, m^{-1}\right) \mid m \in M\right\rangle .
$$

Thus, the natural cover can be obtained from an arbitrary cover $G^{*}$ by a kind of warped Baer sum of $G^{*}$ with itself.

Proof. By Proposition 4.1 and Proposition 4.2 Abelian groups of odd order have natural covers. The definition of $\mathcal{K}\left(G^{*}, 2\right)$ and Theorem 3.2 give the explicit construction of the natural cover starting from an arbitrary one.

We denote the centre of a group $G$ by $Z(G)$. We denote its Frattini subgroup, i.e. the intersection of all maximal subgroups of $G$, by $\Phi(G)$.

We recall that a $p$-group is called special if its centre is equal to its commutator and its Frattini subgroup. A special $p$-group is called extra-special if its centre is cyclic.

Proposition 4.5. Let $p$ be an odd prime number.

(1) The natural cover of $\mathbb{Z} / p \mathbb{Z}$ is just $\mathbb{Z} / p \mathbb{Z}$ itself. 
(2) The natural cover of $(\mathbb{Z} / p \mathbb{Z})^{2}$ is the extra-special group of order $p^{1+2}$ and exponent $p$.

Proof. The first statement follows from Corollary 2.5 .

The Schur multiplier of $G=(\mathbb{Z} / p \mathbb{Z})^{2}$ is $\mathbb{Z} / p \mathbb{Z}$. Hence $\tilde{\mathcal{K}}(G, 2)$ is a nonAbelian group of order $p^{3}$. Such a group is necessarily extra-special. The unique extra-special group $G^{*}$ of order $p^{1+2}$ and exponent $p$ is a cover of $G$. Since $\widetilde{\mathcal{K}}(G, 2)$ is a quotient of $\mathcal{K}\left(G^{*}, 2\right)$ by Theorem 3.2 , the group $\widetilde{\mathcal{K}}(G, 2)$ has exponent $p$. This is enough to identify $\widetilde{\mathcal{K}}(G, 2)$ as the unique extra-special group of order $p^{1+2}$ and exponent $p$.

For applications to algebraic geometry, especially the case $n \geq 3$ is relevant. Using Proposition 1.4, the extension (2) becomes

$$
0 \rightarrow H_{2}(G, \mathbb{Z}) \rightarrow \tilde{\mathcal{K}}(G, n) \rightarrow G^{n-1} \rightarrow 1
$$

Since $n \geq 3$, the group $H_{2}(G, \mathbb{Z})$ lies inside the commutator subgroup of $\tilde{\mathcal{K}}(G, n)$ by Theorem 2.2.

Proposition 4.6. Let $G$ be an Abelian p-group and $n \geq 3$ a natural number.

(1) Unless $G$ is cyclic, the group $\widetilde{\mathcal{K}}(G, n)$ is nilpotent of class 2.

(2) The commutator subgroup of $\widetilde{\mathcal{K}}(G, n)$ is equal to $\mathrm{H}_{2}(G, \mathbb{Z})$ embedded via (7).

(3) The Frattini subgroup $\Phi(\tilde{\mathcal{K}}(G, n))$ is an extension of $H_{2}(G, \mathbb{Z})$ by $\Phi(G)^{n-1}$.

Proof. If $G$ is not cyclic, then $H_{2}(G, \mathbb{Z})$ does not vanish by Schur's theorem, cf. [Ka, Corollary 2.2.12]. Since $\widetilde{\mathcal{K}}(G, n)$ is a central extension of two Abelian groups, it is nilpotent of class at most 2. Since $H_{2}(G, \mathbb{Z})$ lies inside the commutator subgroup of $\widetilde{\mathcal{K}}(G, n)$ by Theorem 2.2 , the group $\widetilde{\mathcal{K}}(G, n)$ is not Abelian.

As $n \geq 3$, the group $H_{2}(G, \mathbb{Z})$ lies inside the commutator subgroup, which shows one inclusion. On the other hand, the quotient of $\widetilde{\mathcal{K}}(G, n)$ by $H_{2}(G, \mathbb{Z})$ is Abelian, showing the other inclusion.

Clearly, $\Phi(\widetilde{\mathcal{K}}(G, n))$ maps onto $\Phi\left(G^{n-1}\right)$ via $(7)$. It is a general fact that $I:=$ $Z\left(G^{*}\right) \cap\left[G^{*}, G^{*}\right]$ is contained in $\Phi\left(G^{*}\right)$. Since $H_{2}(G, \mathbb{Z})$ is contained in $I$ (in fact, they are equal in our case), it follows that $\Phi(\widetilde{\mathcal{K}}(G, n))$ is an extension of $\mathrm{H}_{2}(G, \mathbb{Z})$ by $\Phi\left(G^{n-1}\right) \cong \Phi(G)^{n-1}$.

The structure of the centre of $\widetilde{\mathcal{K}}(G, n)$ is much trickier. In fact, it depends on $n$.

Proposition 4.7. Let $G$ be an Abelian p-group and $n \geq 3$ a natural number. Let $G^{*} \rightarrow G$ be an arbitrary cover of $G$ and denote by $Z$ the image of the centre $Z\left(G^{*}\right)$ inside $G$. 
(1) If the exponent of $G$ divides $n$, then $\widetilde{\mathcal{K}}(G, n)$ is the direct product of $G$ and $\widetilde{\mathcal{K}}(G, n-1)$.

(2) If $p$ does not divide $n$, then the centre of $\widetilde{\mathcal{K}}(G, n)$ is isomorphic to the product of $\mathrm{H}_{2}(G, \mathbb{Z})$ and $\mathcal{K}(Z, n)$.

Proof. We choose a cover $G^{*} \rightarrow G$ with kernel $M$ and define $Z$ as in the statement of the proposition. By Theorem 3.2, the quotient of $\mathcal{K}\left(G^{*}, n\right)$ by $\mathcal{K}(M, n)$ is isomorphic to $\widetilde{\mathcal{K}}(G, n)$.

Suppose that the exponent of $G$ divides $n$. Then we obtain a well-defined injective homomorphism $\Delta$ from $G^{*}$ to $\mathcal{K}\left(G^{*}, n\right)$ that sends $g$ to $(g, \ldots, g)$. Since also $M$ has an exponent which divides $n$, we conclude that the intersection $\Delta(M) \cap \mathcal{K}(M, n)$ is equal to $\Delta(M)$. Hence we obtain $G^{*} / M=G$ as a central subgroup of $\widetilde{\mathcal{K}}(G, n)$. This subgroup maps to a diagonally embedded $G$ inside $\mathcal{K}(G, n)$ (under the map (2)) and hence we can split the induced injective map from $G$ to $\widetilde{\mathcal{K}}(G, n)$. Thus, $G$ is a direct factor of $\widetilde{\mathcal{K}}(G, n)$ and it is easy to see that the quotient is in fact isomorphic to $\widetilde{\mathcal{K}}(G, n-1)$. This also works for $n=2$, but then the quotient of $\widetilde{\mathcal{K}}(G, 2)$ by $G$ is equal to $M$.

To prove the second assertion we now assume that $p$ does not divide $n$. The preimage of the centre of $\widetilde{\mathcal{K}}(G, n)$ in $\mathcal{K}\left(G^{*}, n\right)$ consists of those elements of $\mathcal{K}\left(G^{*}, n\right)$ for which every commutator lies in $\mathcal{K}(M, n)$. Hence this preimage is equal to

$$
\begin{array}{r}
\widetilde{Z}:=\left\{\left(h_{1}, \ldots, h_{n}\right) \in \mathcal{K}\left(G^{*}, n\right) \mid \sum_{i=1}^{n}\left[g_{i}, h_{i}\right]=0\right. \text { for all } \\
\left.\left(g_{1}, \ldots, g_{n}\right) \in \mathcal{K}\left(G^{*}, n\right)\right\} .
\end{array}
$$

Let $\left(h_{1}, \ldots, h_{n}\right) \in \widetilde{Z}$. For $g \in G^{*}$, the element $\left(1, \ldots, g, 1, \ldots, g^{-1}, 1, \ldots\right)$ lies in $\mathcal{K}\left(G^{*}, n\right)$ and we obtain $\left[h_{i}, g\right]=\left[h_{j}, g\right]$ for all $i, j$. In particular, if $h_{i} \in Z\left(G^{*}\right)$ for some $i$, then $h_{i} \in Z\left(G^{*}\right)$ for all $i$.

Assume there exist an element $\vec{h}:=\left(h_{1}, \ldots, h_{n}\right)$ of $\widetilde{Z}$ with $h_{i} \notin Z\left(G^{*}\right)$ for some $i$, say $i=1$. Inside $\left(G^{*}\right)^{n}$ we can write this element as product of $\left(h_{1}, \ldots, h_{1}\right)$ by $\vec{z}:=\left(1, h_{1}^{-1} h_{2}, \ldots, h_{1}^{-1} h_{n}\right)$, where all entries of $\vec{z}$ lie in $Z\left(G^{*}\right)$. The sum over all components of $\vec{z}$ is an element $z^{\prime}$ of $Z\left(G^{*}\right)$. Since $p$ does not divide $n$, there exists a power $z^{\prime \prime}$ of $z^{\prime}$ that is an $n$th root of $z^{\prime}$. We define $\vec{h}^{\prime}:=\left(h_{1} \cdot z^{\prime \prime}, \ldots, h_{1} \cdot z^{\prime \prime}\right)$ and $\vec{z}^{\prime}:=\left(z^{\prime \prime}, \ldots, z^{\prime \prime}\right)^{-1} \vec{z}$. Then $\vec{h}=\vec{h}^{\prime} \cdot \vec{z}^{\prime}$. We arranged $\vec{z}^{\prime}$ in such a way that it lies in $\mathcal{K}\left(G^{*}, n\right)$ and so also $\vec{h}^{\prime}$ lies in $\mathcal{K}\left(G^{*}, n\right)$. Every component of $\vec{h}^{\prime}$ is equal to $h_{1} z^{\prime \prime}$. Since $h_{1} z^{\prime \prime}$ does not lie $\left[G^{*}, G^{*}\right]$ (this group is contained in the centre of $G^{*}$ and we assumed $h_{1} \notin Z\left(G^{*}\right)$ ), also $\left(h_{1} z^{\prime \prime}\right)^{n}$ does not lie in $\left[G^{*}, G^{*}\right]$ (using again the fact that $n$ is coprime to $p)$. Hence $\vec{h}$ does not lie in $\mathcal{K}\left(G^{*}, n\right)$, a contradiction.

We conclude that the centre of $\widetilde{\mathcal{K}}(G, n)$ is the image of $Z\left(G^{*}\right)^{n} \cap \mathcal{K}\left(G^{*}, n\right)$, i.e., we have to compute its quotient by $\mathcal{K}(M, n)$. This group, however, is easily seen to be an extension of $M$ by $\mathcal{K}(Z, n)$. 
Corollary 4.8 (Read's theorem for Abelian groups). Let $G^{*} \rightarrow G$ be a cover of an Abelian group $G$. Then the image $Z$ of the centre $Z\left(G^{*}\right)$ inside $G$ does not depend on the choice of the cover $G^{*}$.

Proof. For a natural number $n \geq 3$ that is coprime to the order of $G$, the centre of $\widetilde{\mathcal{K}}(G, n)$ is a product of $H_{2}(G, \bar{Z})$ and $\mathcal{K}(Z, n)$. Since this centre does not depend on the choice of a cover, also $Z$ is independent of it.

Remark 4.9. In view of the last corollary it seems natural to ask whether $\widetilde{\mathcal{K}}(G, n)$ captures interesting data about all cover groups of a given (not necessarily Abelian) group $G$. For example, Schur's theorem that $\left[G^{*}, G^{*}\right]$ is an invariant of $G$ and does not depend on the choice of the cover $G^{*}$ also follows quite easily from Theorem 3.2.

\section{Fundamental groups}

We now sketch how $\mathcal{K}(G, n)$ and $\tilde{\mathcal{K}}(G, n)$ are connected to fundamental groups of algebraic surfaces and Moishezon's programme to find new invariants for algebraic surfaces. For details and references we refer to [Li].

For complex curves, it is already known since the 19th century, that their fundamental groups classify them up to diffeomorphism. However, although the Italian school classified complex algebraic surfaces of special type in the early 20th century, not much is known about surfaces of general type.

There is an approach towards a finer classification that uses embeddings of surfaces into large projective spaces: Let $X$ be a smooth projective surface and $\mathscr{L}$ a sufficiently ample line bundle on $X$. Then we embed $X$ via $\mathscr{L}$ into some projective space $\mathbb{P}^{N}$. After that we choose a generic codimension three linear subspace in this $\mathbb{P}^{N}$ and consider the projection $\pi$ away from this space. This is a rational map from $\mathbb{P}^{N}$ onto $\mathbb{P}^{2}$. The restriction

$$
f:=\left.\pi\right|_{X}: X \rightarrow \mathbb{P}^{2}
$$

is a finite map, called a generic projection.

We denote by $n$ the degree of $f$ and by $D$ its branch locus. If we know the fundamental group $\pi_{1}\left(\mathbb{P}^{2}-D\right)$ and the monodromy morphism $\psi: \pi_{1}\left(\mathbb{P}^{2}-D\right) \rightarrow \widetilde{S}_{n}$, where $\widetilde{S}_{n}$ denotes the symmetric group on $n$ letters, we can reconstruct $X$. Thus, if we could extract invariants from these fundamental groups we would get a much finer classification of algebraic surfaces. However, these groups $\pi_{1}\left(\mathbb{P}^{2}-D\right)$ are huge and may have a rather complicated structure, although a conjecture of Teicher states that they are almost-solvable.

Also this was known for some time, but it could not be used effectively since it was too difficult to compute these fundamental groups. However, the braid group techniques introduced by Moishezon in [Mo], and refined later on by Teicher and others, made it possible to compute these groups $\pi_{1}\left(\mathbb{P}^{2}-D\right)$ in many cases. 
One such invariant for $X$ depending on the choice of an embedding of $X$ into projective space is the fundamental group of the Galois closure of this generic projection: To a generic projection $f$ of degree $n$ we associate its so-called Galois closure

$$
X_{\text {gal }}:=\overline{\left\{\left(x_{1}, \ldots, x_{n}\right) \mid x_{i} \neq x_{j}, f\left(x_{i}\right)=f\left(x_{j}\right)\right\}} \subseteq X^{n} .
$$

This turns out to be a smooth projective surface. In most cases it is of general type.

Apart from their connection with generic projections, there is another reason why Galois closures are interesting: Using Galois closures of generic projections gives one of the few known ways to construct series of surfaces of general type with positive index, i.e., the Chern numbers of $X_{\text {gal }}$ fulfil $c_{1}^{2}>2 c_{2}$. For some time it was believed that surfaces of general type with positive index should have infinite fundamental groups. The first counter-examples to this conjecture were given by Moishezon and Teicher via computing fundamental groups of Galois closures of generic projections from $X=\mathbb{P}^{1} \times \mathbb{P}^{1}[\mathrm{MoTe}]$.

Hence, determining $\pi_{1}\left(X_{\mathrm{gal}}\right)$ is interesting from the point of view of fundamental groups of surfaces of general type. Also, these groups should give new invariants of $X$ as they occur as certain naturally defined subquotients of $\pi_{1}\left(\mathbb{P}^{2}-D\right)$. In [Li] we partly simplified the calculations of [MoTe] which led to $\widetilde{\mathcal{K}}(G, n)$ :

We fix a generic projection $f: X \rightarrow \mathbb{P}^{2}$ of degree $n$ and denote by $X_{\text {gal }}$ its associated Galois closure $f_{\text {gal }}: X_{\text {gal }} \rightarrow \mathbb{P}^{2}$. We fix a generic line in $\mathbb{P}^{2}$ and denote its complement by $\mathbb{A}^{2}$. Then we denote the inverse images of $f^{-1}\left(\mathbb{A}^{2}\right)$ and $f_{\text {gal }}{ }^{-1}\left(\mathbb{A}^{2}\right)$ by $X^{\text {aff }}$ and $X_{\text {gal }}^{\text {aff }}$, respectively. Since $f$ is generic the Galois group of $X_{\text {gal }}$ over $\mathbb{P}^{2}$ is the whole symmetric group $\subseteq_{n}$. This group acts on $X_{\text {gal }}$ and $X_{\text {gal }}^{\text {aff }}$ and we can form the quotient

$$
\begin{aligned}
& X_{\mathrm{gal}} / \mathfrak{S}_{n-1} \cong X, \\
& X_{\mathrm{gal}}^{\mathrm{aff}} / \mathfrak{S}_{n-1} \cong X^{\mathrm{aff}} .
\end{aligned}
$$

There are $n$ distinct embeddings of $\subseteq_{n-1}$ into $\widetilde{S}_{n}$ yielding $n$ distinct isomorphisms and $n$ distinct induced maps on fundamental groups, all of which are surjective:

$$
\begin{aligned}
& \pi_{1}\left(X_{\text {gal }}\right) \rightarrow \pi_{1}(X), \\
& \pi_{1}\left(X_{\text {gal }}^{\text {aff }}\right) \rightarrow \pi_{1}\left(X^{\text {aff }}\right) .
\end{aligned}
$$

Combining these $n$ homomorphisms, we obtain a map from $\pi_{1}\left(X_{\text {gal }}\right)$ to $\pi_{1}(X)^{n}$, and similarly for $\pi_{1}\left(X_{\text {gal }}^{\text {aff }}\right)$. The following result determines the images of these maps.

Theorem 5.1. There exist surjective homomorphisms

$$
\begin{aligned}
& \pi_{1}\left(X_{\text {gal }}\right) \rightarrow \mathcal{K}\left(\pi_{1}(X), n\right), \\
& \pi_{1}\left(X_{\text {gal }}^{\text {aff }}\right) \rightarrow \mathcal{K}\left(\pi_{1}\left(X^{\text {aff }}\right), n\right) .
\end{aligned}
$$


The arguments in the proof of Theorem 5.1 can be formalised in such a way that the result remains true for étale fundamental groups and generic projections defined over arbitrary algebraically closed fields of characteristic $\neq 2,3$. Of course, one has to modify the statement for $\pi_{1}\left(X_{\text {gal }}^{\text {aff }}\right)$ over fields of positive characteristic a little bit since the affine plane is then no longer simply-connected.

Over the complex numbers the algorithm of Zariski and van Kampen provides us with a presentation of fundamental groups of complements of curves in $\mathbb{A}^{2}$ or $\mathbb{P}^{2}$. Applying it to the branch curve $D$ of $f$ we find the fundamental group $\pi_{1}\left(X_{\text {gal }}^{\text {aff }}\right)$ as a subquotient of $\pi_{1}\left(\mathbb{A}^{2}-D\right)$. Combining this presentation with Theorem 5.1 we obtain

Theorem 5.2. There exists a surjective homomorphism

$$
\pi_{1}\left(X_{\text {gal }}^{\text {aff }}\right) \rightarrow \widetilde{\mathcal{K}}\left(\pi_{1}\left(X^{\mathrm{aff}}\right), n\right) .
$$

The group $\pi_{1}\left(X_{\mathrm{gal}}\right)$ is a quotient of $\pi_{1}\left(X_{\mathrm{gal}}^{\mathrm{aff}}\right)$ by a cyclic central subgroup.

In all known examples, where the generic projection was defined via a sufficiently ample line bundle, the map of Theorem 5.2 is in fact an isomorphism.

This suggests to use Galois closures of generic projections to construct algebraic surfaces with interesting fundamental groups. For example, starting from a surface with Abelian fundamental group, iterated Galois closures should produce surfaces with nilpotent fundamental groups of large class. Another project would be to obtain new surfaces with fundamental groups that are not residually finite.

Whether the map of Theorem 5.2 is an isomorphism in all cases or at least for large class of surfaces or generic projections is not clear at the moment, although this is true in all known examples. In any case, we have a quotient of the group we are interested in and the appearance of covering groups in connection with these fundamental groups is quite surprising. Therefore, it is indispensable to have a better understanding of $\widetilde{\mathcal{K}}(-, n)$ to comprehend these fundamental groups.

\section{References}

[Br] K. S. Brown, Cohomology of groups. Grad. Texts in Math. 87, Springer-Verlag, New York 1982. Zbl 0584.20036 MR 0672956

[Ka] G. Karpilovsky, The Schur multiplier. London Math. Soc. Monogr. 2, Oxford University Press, Oxford 1987. Zbl 0619.20001 MR 1200015

[Li] C. Liedtke, On fundamental groups of Galois closures of generic projections. Bonner Math. Schriften 367, Univ. Bonn, Mathematisches Institut, Bonn 2004.

Zbl 1095.14013 MR 2206239 
[Mo] B. Moishezon, Stable branch curves and braid monodromies. In Algebraic geometry (Chicago, 1980), Lecture Notes in Math. 862, Springer-Verlag, Berlin 1981, 107-192. Zbl 0476.14005 MR 0644819

[MoTe] B. Moishezon and M.Teicher, Simply-connected algebraic surfaces of positive index. Invent. Math. 89 (1987), 601-643. Zbl 0627.14019 MR 0903386

Received September 13, 2007; revised November 20, 2007

C. Liedtke, Mathematisches Institut, Heinrich-Heine-Universität, 40225 Düsseldorf, Germany

E-mail: liedtke@math.uni-duesseldorf.de 